\title{
COMPUTATION OF VORTEX SHEDDING AND RADIATED SOUND FOR A CIRCULAR CYLINDER
}

\author{
Jared S. Cox \\ The George Washington University, JIAFS \\ Hampton, Virginia \\ Kenneth S. Brentner \\ NASA Langley Research Center \\ Hampton, Virginia
}

\author{
Christopher L. Rumsey \\ NASA Langley Research Center \\ Hampton, Virginia
}

\author{
Bassam A. Younis \\ City University \\ London, England
}

\begin{abstract}
The Lighthill acoustic analogy approach combined with Reynolds-averaged Navier Stokes is used to predict the sound generated by unsteady viscous flow past a circular cylinder assuming a correlation length of ten cylinder diameters. The twodimensional unsteady flow field is computed using two NavierStokes codes at a low Mach number over a range of Reynolds numbers from 100 to 5 million. Both laminar flow as well as turbulent flow with a variety of eddy viscosity turbulence models are employed. Mean drag and Strouhal number are examined, and trends similar to experiments are observed. Computing the noise within the Reynolds number regime where transition to turbulence occurs near the separation point is problematic: laminar flow exhibits chaotic behavior and turbulent flow exhibits strong dependence on the turbulence model employed. Comparisons of far-field noise with experiment at a Reynolds number of 90,000, therefore, vary significantly, depending on the turbulence model. At a high Reynolds number outside this regime, three different turbulence models yield self-consistent results.
\end{abstract}

\section{INTRODUCTION}

The sound generated by the unsteady viscous flow over a circular cylinder is representative of several bluff body flows found in engineering applications (e.g., automobile antenna noise, aircraft landing gear noise, etc.). Although extensive experimental studies have been performed on circular cylinder flows (see, e.g., Morkovin ${ }^{1}$ ), many questions about the physical processes that occur still exist, particularly at higher Reynolds numbers. Numerically, many computational fluid dynamics (CFD) studies have also been performed, but primarily only at low Reynolds numbers with purely laminar flow. The ability to accurately compute cylinder noise across a broad range of Reynolds numbers with numerical methods would enhance the understanding of bluff body noise generation mechanisms.

A complete understanding of circular cylinder flow is particularly elusive because transition from laminar to turbulent flow occurs in a distinct succession over an enormous range of Reynolds numbers, and each transition state is sensitive to extremely small disturbances. ${ }^{2}$ These disturbances, such as free stream turbulence and surface roughness, can significantly alter the range of Reynolds numbers over which each transition state occurs. Especially at Reynolds numbers at and above roughly
100,000 , experiments can show widely different behavior due to differences in the experimental flow conditions.

The flow around a circular cylinder is often characterized into three distinct flow regimes: subcritical, supercritical, and transcritical $^{3}$ ). Subcritical flow indicates purely laminar boundary layer separation. In this regime, regular vortex shedding at a Strouhal number of about 0.2 is observed over a range of Reynolds numbers from roughly 200 to 100,000 . The supercritical regime, from Reynolds numbers of roughly 100,000 to 4 million, is characterized by either a dramatic rise in the Strouhal number or else a loss of organized vortex shedding altogether. $^{4,5}$ Also, the wake is noticeably narrower and the forces are much smaller in magnitude. ${ }^{6}$ It is somewhere in this regime that transition to turbulence begins to occur on the body at or near the point of separation. In the transcritical regime, above a Reynolds number of roughly 4 million, periodic vortex shedding re-establishes at a higher Strouhal number of $0.26-0.30 .^{3,5,7}$ The cylinder now experiences fully turbulent boundary layer separation and higher force coefficients than in the supercritical regime. ${ }^{6}$

Any attempt to numerically model circular cylinder flow is complicated by the fact that the flow above a Reynolds number of around 180 is three-dimensional, ${ }^{8,9}$ raising doubts about the applicability of two-dimensional simulations. Additionally, transition occurs off-body in the wake or shear layer at Reynolds numbers between roughly 200 and the supercritical regime. ${ }^{2}$ Without performing very expensive direct numerical simulations (DNS), this behavior is not captured by numerical methods that solve the Navier-Stokes equations on typical grids used for aerodynamic analysis. This deficiency may or may not be important at lower Reynolds numbers, depending on how far behind the cylinder transition occurs and what feature of the flow is of interest. But it certainly has an adverse effect at higher Reynolds numbers for which transition occurs at or near the separation point on the cylinder. For Reynolds numbers at and above the supercritical regime, Reynolds-averaging with the use of a turbulence model is one way to introduce the important effect of turbulence into a numerical simulation. However, without an accurate built-in transition model, it is difficult, if not impossible, to model the important effects of transition, particularly when it occurs on or near the body. It is not surprising, then, that most numerical studies of flow around a circular cylinder have focused primarily 
on low Reynolds number flows less than about 1000.

At Reynolds numbers of roughly 200 or less, many researchers have successfully computed the Strouhal number and mean drag over a circular cylinder (see, for example references, 10-14). At higher Reynolds numbers, however, two-dimensional numerical methods cannot predict the lift and drag forces accurately, due to the increasingly prominent three-dimensionality of the the real flow field. ${ }^{8,9}$ Nonetheless, it is still important to try to understand and characterize the capabilities and limitations of existing two-dimensional numerical methods at higher Reynolds numbers (where most bluff-body noise sources of interest occur), since these methods may yield a deeper insight into the physics at a relatively low cost.

In this work, an investigation is made into the ability to numerically predict the aerodynamic properties of a circular cylinder across a range of Reynolds numbers from 100 to 5 million. The unsteady viscous flow field is computed by two different Reynolds-averaged Navier Stokes (RANS) flow solvers with a variety of turbulence models, as well as at lower Reynolds numbers using the laminar Navier-Stokes equations. The computations are two-dimensional and time-accurate. The flow-field computations are emphasized because they are the most challenging aspect of the noise prediction problem.

The noise is calculated with an acoustic prediction code that uses the calculated unsteady surface pressures as input. The acoustic code uses an advanced acoustic analogy integral formulation developed by Farassat. ${ }^{15}$ In this paper, results are presented at a single observer location for each of the flow-field computations. The noise predictions are compared with the experiment of Revell. ${ }^{16}$ A companion paper by the authors ${ }^{17}$ deals more fully with the acoustic aspects of the problem.

No attempt is made in this paper to predict broadband noise. To do this, it maybe necessary to resolve the small-scale structures and fluctuations using DNS, rather than model the global effects of turbulence using an effective eddy viscosity as is done currently using RANS.

\section{APPROACH}

\section{Flow Field Calculation}

Two unsteady RANS codes (CFL3D and CITY3D) are utilized in this work. In the context of these codes, Reynoldsaveraging denotes averaging over a time interval which is very long relative to the maximum period of the turbulent velocity fluctuations, but shorter than the vortex shedding period of the cylinder. A turbulence model must be employed to account for the effect of the Reynolds stress tensor that arises from the Reynolds averaging. In the current work, either a one- or twoequation model is employed which makes use of the Boussinesq eddy-viscosity assumption to compute the Reynolds stress tensor as the product of an eddy viscosity and the mean strain-rate tensor.

The first code, CFL3D, ${ }^{18}$ is a 3 -D thin-layer compressible Navier-Stokes code which employs the finite volume formulation in generalized coordinates. It employs upwind-biased spatial differencing for the convective and pressure terms, and central differencing for the viscous terms. It is globally second order accurate in space, and employs Roe's flux difference splitting. ${ }^{19}$
The code is advanced implicitly in time using 3-factor approximate factorization. ${ }^{18}$ Temporal subiterations with multigrid are employed to reduce the linearization and factorization errors. For the current study, CFL3D was run in a 2-D time-accurate mode which is second-order accurate in time. Viscous derivative terms are turned on in both coordinate directions, but the cross-coupling terms are neglected as part of the thin-layer assumption.

CFL3D has a wide variety of turbulence models available, including zero-equation, one-equation, and two-equation (linear as well as nonlinear). For the current study, the code was run either laminar only (i.e., no Reynolds averaging), or else employed a turbulence model using the Boussinesq eddy-viscosity assumption. The three turbulence models tested were the SpalartAllmaras (S-A) one-equation model, ${ }^{20}$ the shear stress transport (SST) two-equation $k-\omega$ model of Menter, ${ }^{21}$ and the twoequation $k-\epsilon$ model of $\mathrm{Abid} .^{22}$ The S-A model is widely used throughout the U.S. aerospace community for the prediction of steady attached and separated flows, and was also used by Rumsey et al. ${ }^{18}$ to compute the unsteady self-excited shock-induced flow oscillations about a biconvex airfoil. The SST model is a blend of the $k-\omega$ and $k-\epsilon$ turbulence equations, with an additional correction to the eddy viscosity to account for the transport of the principal turbulent shear stress. It has been demonstrated to yield good results for a wide variety of steady separated turbulent aerodynamic flows, ${ }^{23}$ but its capabilities for unsteady flows remains relatively untested. The Abid $k-\epsilon$ model, while similar to the more widely-used Jones-Launder version, ${ }^{24}$ remains relatively untested for unsteady flows.

When turbulence models are employed, the CFL3D code integrates the field equations to the wall, so that grids with minimum spacings on the order of $\Delta y+=1$ must be employed to ensure adequate resolution in the laminar sub-layer. Turbulence, in the form of an eddy-viscosity, is produced as a result of strain in the boundary layer of the circular cylinder. The magnitude of the eddy viscosity produced is also a function of the Reynolds number. Therefore, the field equation models "transition" on their own; depending on the local strain and Reynolds number, the production of eddy viscosity may or may not be large enough to produce enough turbulence to "trip" the boundary layer to become turbulent. In practice, at a low Reynolds number on the order of 100,000 , the circular cylinder boundary layer does not "trip" until approximately 90 degrees from the stagnation point, near where the boundary layer separates. At higher Reynolds numbers of 1 million or more, the boundary layer transitions to turbulence very near to the stagnation point. Hence, the boundary layer is fully turbulent when separation occurs.

The second code, CITY $3 \mathrm{D},{ }^{25}$ is a finite-volume code for the solution of the incompressible, 3-D Navier-Stokes equations in generalized coordinates. A pressure-correction technique is used to satisfy mass and momentum conservation simultaneously. Temporal and spatial discretizations are first- and thirdorder accurate, respectively. The turbulence model used in this study is the $k-\epsilon$ model modified as described in reference 25 to account for the effects of superimposing organized mean-flow periodicity on the random turbulent motions. The modification takes the form of an additional source to the $\epsilon$ equation which 


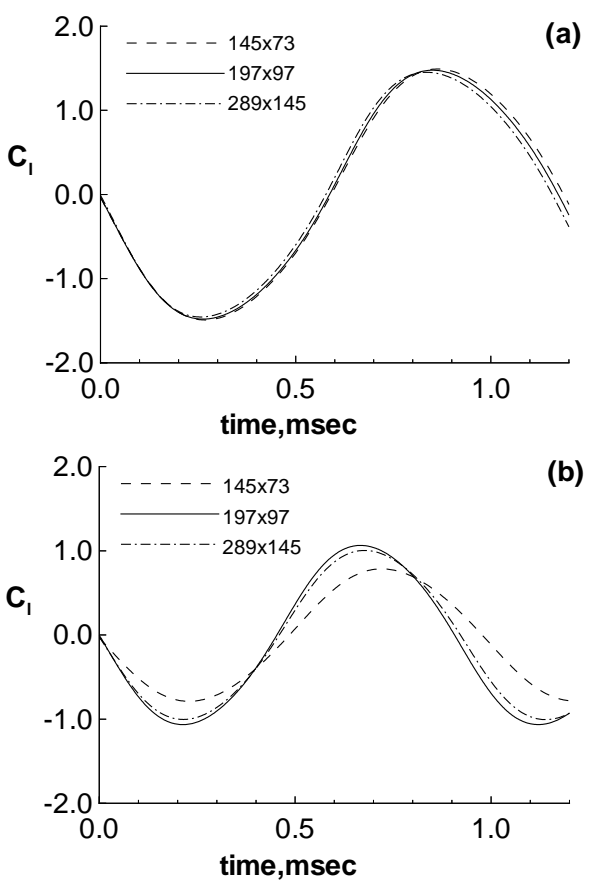

Figure 1. Comparison of predicted $C_{l}$ time histories for different grid resolutions. (a) laminar $R e=1000$; (b) SST $R e=5$ million.

represents the direct energy input into the turbulence spectrum at the Strouhal frequency. CITY3D does not integrate the turbulence equations to the wall, but employs wall functions which presume a turbulent boundary layer profile governed by the lawof-the-wall.

Previous studies ${ }^{18,26}$ have addressed the effect of time step for unsteady flows over circular cylinders and airfoils. These studies indicated that on the order of 500 time steps per cycle is generally sufficient to insure adequate temporal resolution, although this number is a function of oscillation frequency. In the current study with CFL3D, a nondimensional time step of 0.05 (nondimensionalized by cylinder diameter and freestream speed of sound) is employed, yielding between 300 - 600 steps per shedding cycle, depending on the case. Although not shown, this time step has been shown to yield results that do not change appreciably with further time step refinement (for example, increasing to over 1000 steps per cycle alters the computed Strouhal number by less than one percent for laminar flow at $R e=1000$ ). CITY3D utilized a time step corresponding to 470 steps per shedding cycle.

The effect of grid size for the CFL3D code is shown in figures 1 and 2 . In the grid study, three grid sizes are employed: $289 \times 145,197 \times 97$, and $145 \times 73$. All grids are O-grids, have circumferential clustering in the wake region, and are stretched radially to a far-field extent of 20 diameters. For Reynolds numbers less than or equal to 100,000 , the minimum spacing at the cylinder is 0.00005 diameters, while for higher Reynolds numbers the minimum spacing used is 10 times finer. The $145 \times 73$ coarse

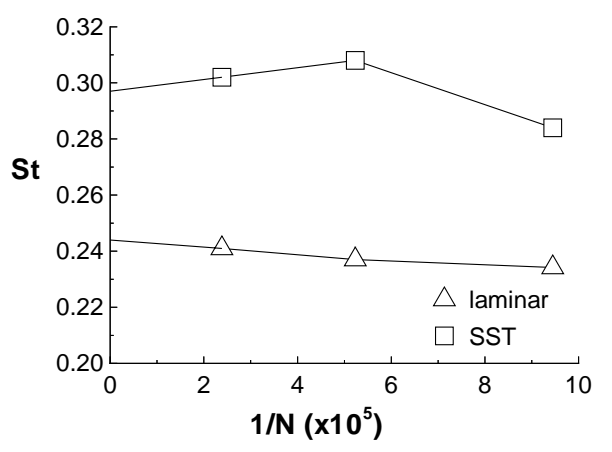

Figure 2. Strouhal number vs. grid density.

grid consists of every other point from the $289 \times 145$ fine grid. The CFL3D code is run with freestream Mach number $M=0.2$. The code's results are nondimensional, but dimensional times are shown here assuming a cylinder diameter of $D=0.019 \mathrm{~m}$ and freestream speed of sound of $340 \mathrm{~m} / \mathrm{s}$. In figure 1 (a), the lift coefficient variation with time is shown for laminar flow at $R e=1000$ for the three grid densities. Very little variation is evident between the three grids. Figure 1 (b) shows Reynoldsaveraged turbulent flow results at $R e=5$ million using the SST turbulence model. In this case, there is little difference between the medium and fine grids, but the coarse grid shows greater variation.

Figure 2 shows Strouhal number for both the laminar and turbulent cases as a function of $1 / \mathrm{N}$, where $\mathrm{N}$ is the total number of grid points. For a globally second-order spatially accurate algorithm, the variation should be linear on sufficiently fine grids. In this case, we assume that the medium and fine grids are sufficiently fine, and extrapolate to an infinite grid size to get an idea of the discretization error on each of the finite grids. For both the laminar and turbulent cases, the error in computed Strouhal number on the medium $(197 \times 97)$ grid is less than 4 percent from an infinite grid, whereas the fine grid $(289 \times 145)$ is less than 2 percent. The $197 \times 97$ grid is used for all the remaining CFL3D computations in this paper. The CITY3D code uses a different grid of size $148 \times 102$. Because it employs a wall function boundary condition, the minimum spacing at the cylinder surface of 0.02 diameters is considerably larger than the grids used by CFL3D.

The effect of grid extent is not addressed here. However, a previous study by Rumsey et al. ${ }^{12}$ indicated a relatively small dependence of computed Strouhal number on the far field grid extent (approximately 2 - 3 percent error from an extrapolated infinite-grid-extent solution) when a 20 diameter grid extent was employed. CITY3D uses a grid extent of 48 diameters.

\section{Acoustic Calculation}

The unsteady flow-field calculation from CFL3D or CITY3D is used as input into an acoustic prediction code WOPWOP ${ }^{27}$ to predict the near- and far-field noise. WOPWOP is a rotor noise prediction code based upon Farassat's retarded-time formulation $1 \mathrm{~A},{ }^{15}$ which is a solution to the Ffowcs Williams Hawkings (FW-H) equation ${ }^{28}$ with the quadrupole source neglected. Details of the acoustic prediction methodology are given 
in reference 17 .

Unlike the CFD calculations, the integration performed for the acoustic calculation is over a three-dimensional cylinder that is translating in a stationary fluid. For the predictions in this paper, we assume that the surface pressures are constant along the span at any source time. Experiments and computational work (e.g., references 1,6,29,30) have shown that vortex shedding is intrinsically three-dimensional and the shedding is correlated only over some length (typically $<10 D$ ). To account for the likelihood of partially correlated vortex shedding, the noise predictions use a cylinder length of 10 diameters. Truncating the cylinder for noise predictions in no way models the complicated, three-dimensional flow physics, but it does avoid a substantial overprediction that would result from a much longer cylinder length. ${ }^{17}$

(a)

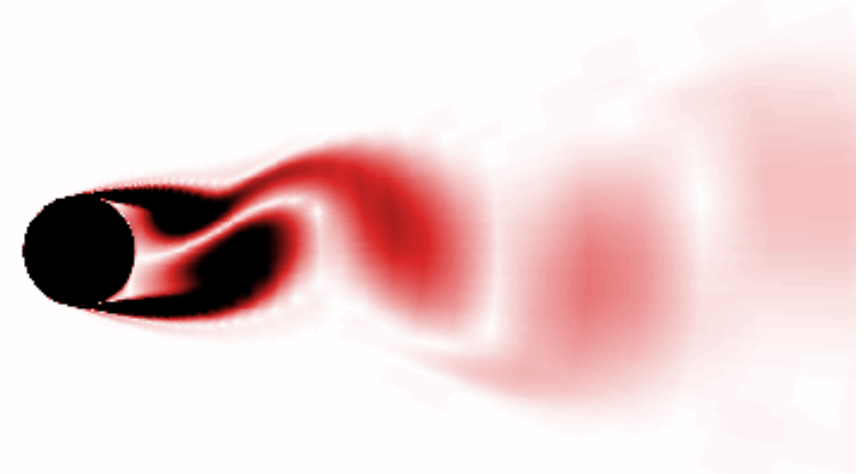

(b)

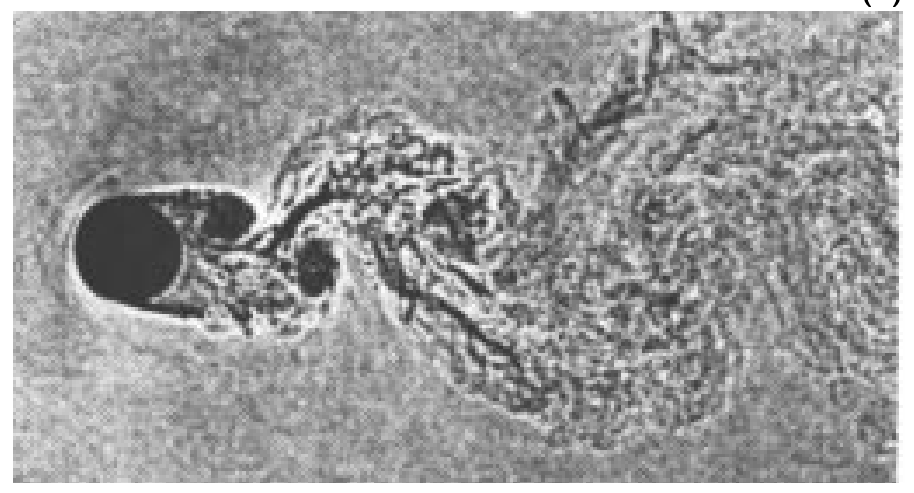

Figure 3. Flow field visualization. (a) vorticity magnitude contours: SST $R e=90,000$; (b) multiple spark shadowgraph: $:^{31}$ $R e=140,000$.

\section{RESULTS}

\section{Flow Field Prediction}

CFD calculation of the flow field physics has proven to be the most challenging part of the problem of sound prediction for the circular cylinder. This is especially evident for Reynolds numbers where the boundary layer is transitioning from laminar to turbulent flow in and near the supercritical regime. Flow field computations are made assuming fully laminar flow at $R e=100$, 200, 1000, 10,000 and 90,000. Turbulent computations are made at $R e=90,000$ and 5 million with the S-A, SST, and $k^{-}$ $\epsilon$ turbulence models. Turbulent results were also obtained at $R e=90,000$ using the modified $k-\epsilon$ model in CITY3D. Figure 3 shows vorticity contours at an instant in time for the SST turbulence model. This is compared qualitatively with a shadowgraph photograph $^{31}$ for a similar flow. This comparison is illustrative in showing that the CFD calculation is able to produce the von Karman vortex street with similar shape and dissipation, i.e., two very distinct vortices in the near wake, followed by larger, more diffuse, vortices downstream.

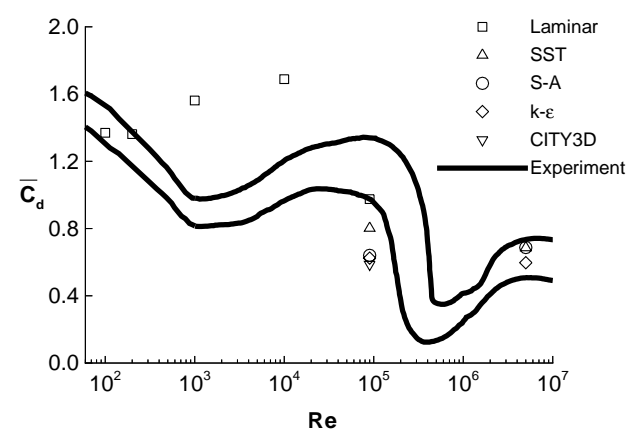

(a)

(b)

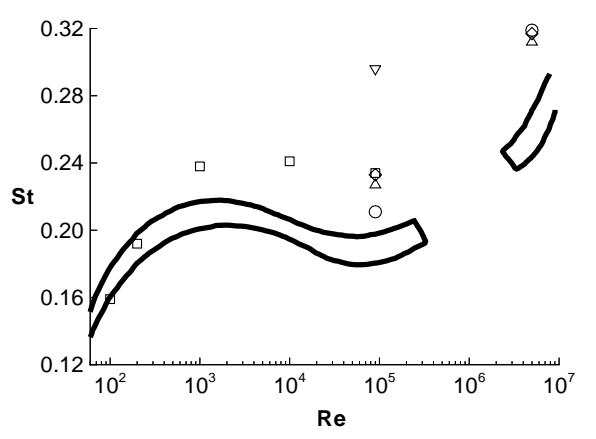

Figure 4. Comparison of predicted $\overline{\boldsymbol{C}}_{d}$ and $\boldsymbol{S t}$ with experiment. (a) $\bar{C}_{d}$ vs. $R e$; (b) $S t$ vs. $R e$.

An approximate composite of experimental data ${ }^{1,5,32}$ for mean drag $\left(\bar{C}_{d}\right)$ and shedding frequency $(S t)$ is shown as a band between two thick lines in figure 4 . At the critical Reynolds number a little above $R e=100,000$ the drag crisis occurs, where the mean drag coefficient drops significantly $\left(\bar{C}_{d} \approx 1.2\right.$ to $\left.\bar{C}_{d} \approx .3\right)$ This is the start of the supercritical Reynolds number regime. Any increase in free stream turbulence or surface roughness significantly alters the Reynolds number where the drag crisis occurs (see reference 5, p. 664).

At Reynolds numbers slightly beyond 100,000 the Strouhal number either increases dramatically or else regular vortex shedding ceases altogether. ${ }^{4,5}$ This dramatic change in the character of the shedding is indicated by the absence of experimental data in figure $4(\mathrm{~b})$. Around $R e \approx 4$ million, regular vortex shedding reappears. ${ }^{3,5,7}$ 

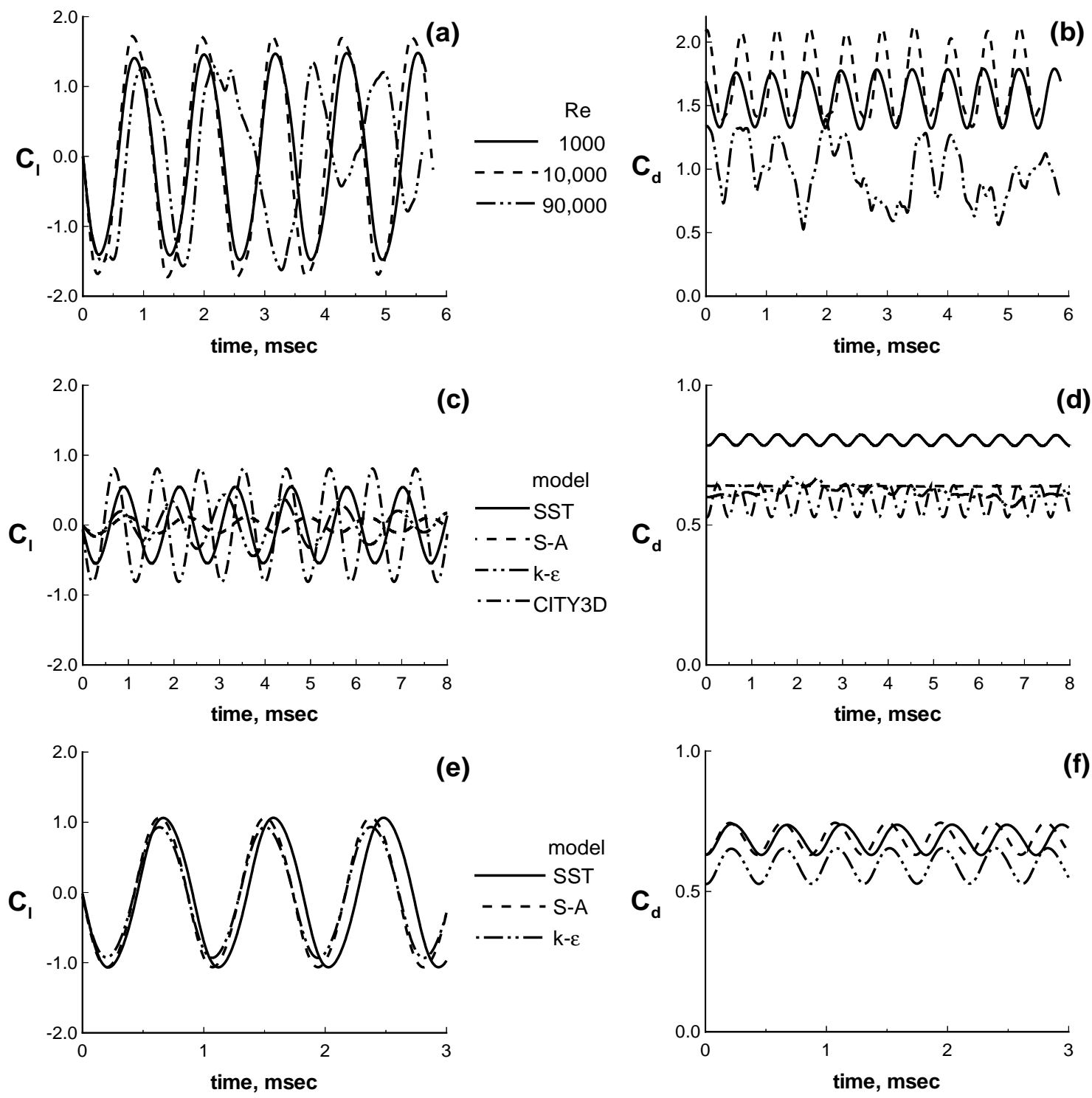

Figure 5. Comparison of predicted $C_{l}$ and $C_{d}$ time histories for $M=0.2$ flow past a 2-D circular cylinder. (a) laminar $C_{l}$ predictions; (b) laminar $C_{d}$ predictions; (c) turbulent $R e=90,000 C_{l}$ predictions; (d) turbulent $R e=90,000 C_{d}$ predictions; (e) turbulent $R e=5$ million $C_{l}$ predictions; (f) turbulent $R e=5$ million $C_{d}$ predictions. Note: the vertical scale is not the same for all $C_{d}$ plots. 
The CFD results are indicated by the symbols in figure 4 . First, note that CFL3D predicts both Strouhal number and drag in agreement with experimental data for $R e=100$ and 200. This is near the Reynolds number range where the flow is known to be two-dimensional. For $R e=1000$ and 10,000, both the drag and Strouhal number are overpredicted; this is most likely due to three-dimensional effects. ${ }^{8}$ The laminar $R e=90,000$ prediction falls within the experimental band. This is possibly fortuitous, since this laminar solution is quite erratic (see figure 5 (a) and (b)).

The erratic behavior of the laminar solution with increasing Reynolds number is consistent with what has been observed by others. $^{33,34}$ It is believed to be a reflection of the increasingly turbulent nature of the flow, but it is probably in part a numerical artifact because the grid and time step are insufficient to resolve the turbulent eddies. The turbulent predictions at $R e=90,000$ exhibit an early drag crisis consistent with experiment for increased free stream turbulence. The turbulent predictions for $R e=5$ million fit very nicely within the experimental band. All computations for $R e \geq 1000$ predicted Strouhal numbers higher than experiment, but the computed Strouhal number dependence on Reynolds number is similar to the experimental trend. Notice the CITY3D Strouhal number is quite high for the $R e=90,000$ case. This is possibly due to the fact that the CITY3D code uses wall functions for the velocity profile and therefore has a fully turbulent boundary layer, more consistent with the $R e=5$ million cases with CFL3D.

Figures 5 (a)-(f) show portions of the time histories of $C_{l}$ and $C_{d}$ for each of the cases, except the low Reynolds number calculations which were simply used to validate the CFL3D code. The time dimensions are obtained by assuming a cylinder diameter $D=0.019 \mathrm{~m}$ and freestream speed of sound $340 \mathrm{~m} / \mathrm{s}$. The laminar cases in figures 5 (a) and (b) are fairly similar in fluctuating $C_{l}$ amplitude; however, the $R e=90,000$ case is quite irregular and only a representative sample is shown. Both the fluctuating and $\bar{C}_{d}$ values of the laminar solutions vary considerably with Reynolds number. Figures 5 (c) and (d) illustrate the difficulties experienced by the CFD computations near the critical Reynolds number. There are large differences between all of the turbulence models. S-A predicts a small $C_{l}$ fluctuation and an almost flat $C_{d}$ fluctuation. The $k-\epsilon$ model exhibits an additional low frequency $C_{l}$ peak-to-peak fluctuation and the associated $C_{d}$ is quite irregular. The SST and CITY3D solutions have a high level of regularity, but do not agree with each other. Because each of the CFL3D turbulence models "transitions" very near the separation point, slight variations between the models are likely to cause significant differences in the solutions. Note that these turbulent computations have lower $C_{l}$ fluctuation amplitudes than laminar computations, in general agreement with the experimental observation that higher levels of turbulence yield lower lift amplitudes. ${ }^{35}$ Once the boundary layer is fully turbulent ( $R e=5$ million cases) the three turbulence models agree very well, as shown in figures 5 (e) and (f).

\section{Acoustic Prediction}

To test the coupling of the CFD and acoustic codes, we choose to predict the noise generated by flow past the circular cylinder for an observer position at a location $90 \mathrm{deg}$ from the

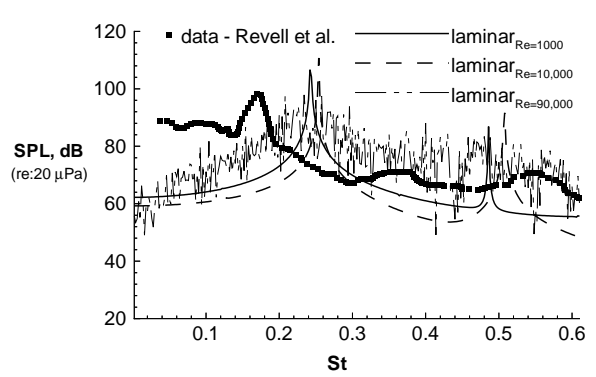

(a)

(b)

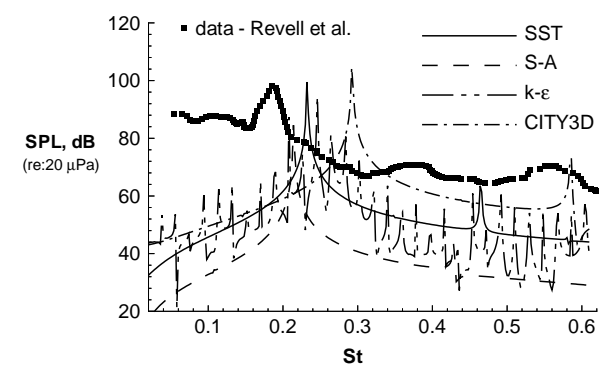

(c)

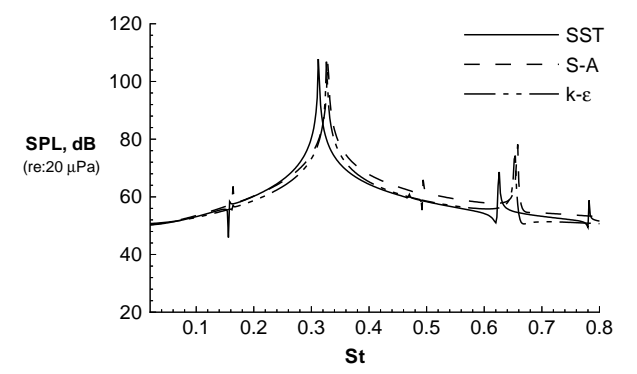

Figure 6. Comparison of predicted and measured sound pressure level for a microphone located $128 D$ away from the cylinder and at a $90 \mathrm{deg}$ angle to the freestream flow. (a) laminar predictions compared to $R e=89,000$ data; $^{16}$ (b) turbulent $R e=90,000$ predictions compared to $R e=89,000$ data: $^{16}$ (c) turbulent $R e=5$ million predictions

freestream direction and 128 cylinder diameters away from the cylinder. This corresponds to a microphone location in the experiment ( $R e=89,000$ and $M=.2$ ) conducted by Revell et al. ${ }^{16}$ The predicted acoustic spectra for each of the CFD inputs are shown in figure 6 along with the experimental data. It is very important to note that the only relevant information from the computations is at the peaks of the fundamental and 1st harmonic. All of the "skirting" is simply a numerical artifact related to having a non-integer multiple of periods contained within the lift vs. time sample chosen for analysis. One period of surface pressure data (repeated as necessary) was used to predict the noise. (Because the loading time histories were irregular, approximately 62 cycles of input data were used in the noise calculation of the laminar $R e=90,000$ case, and approximately 7 cycles were used for the $k-\epsilon R e=90,000$ case.) 
For these predictions, a cylinder length of 10 diameters $(0.19$ $\mathrm{m})$ was chosen to approximate the effect of only partially correlated vortex shedding. This length was chosen because it represents an upper bound on the correlation length found in many experiments. A more detailed analysis of the acoustic predictions, including the effect of correlation length, is contained in Brentner et al. ${ }^{17}$

The amplitude of the noise predicted by the SST turbulence model matches well with experiment (figure 6 (b)). This is, however, a strong function of the choice of correlation length. Using the same correlation length, both S-A and $k-\epsilon$ underpredict the noise, while CITY3D overpredicts the noise. The chaotic laminar flow solution at $R e=90,000$ produces a noise level in good agreement with experiment, while the more regular lower Reynolds number results predict noise levels that are too high (figure $6(\mathrm{a})$ ). It is important to note that the primary driver for the noise is the fluctuating lift, therefore any overprediction of lift amplitude will overpredict the noise and likewise an underprediction of lift will underpredict the noise. ${ }^{17}$ All predicted Strouhal numbers are larger than the Strouhal number in Revell's experiment; ${ }^{16}$ however, this experimental value is in the lower range of experimental data shown in figure 4. Finally, figure 6 (c) shows noise predictions at $R e=5$ million. Again, the inputs from various turbulence models yield consistent acoustic results for this Reynolds number at which the cylinder boundary layer is fully turbulent.

\section{CONCLUDING REMARKS}

The ability to compute the noise generated by the unsteady flow over a circular cylinder hinges primarily on the accuracy of the CFD numerical method in modeling the physics. Because most of today's CFD codes still lack sophisticated transitionprediction models, this proves to be quite a difficult task for the range of Reynolds numbers where transition plays a key role. Also, two-dimensional numerical modeling misses key features of the inherently three-dimensional flow, particularly partiallycoherent shedding, thus requiring a simulated correlation length for noise predictions.

In spite of these shortcomings, the two-dimensional simulations presented here have identified some of the underlying mechanisms which cause the different behaviors of the cylinder flow as it progresses from the subcritical to the supercritical and transcritical regimes. In this paper, the effect of Reynolds number and turbulence modeling on the computed circular cylinder flow field were explored with regard to the far-field noise produced by the unsteady flow.

Computations using laminar flow Navier-Stokes equations produce regular vortex shedding at lower Reynolds numbers, but become increasingly chaotic as the Reynolds number approaches the supercritical range. This reflects the increasingly turbulent character of the flow; however, the grids and time steps employed in this study are far too coarse to resolve the turbulent eddies sufficiently (as in DNS), so the randomness exhibited by the computations is probably more a numerical artifact rather than a representation of any physics. Nonetheless, the noise predicted by the laminar solution at $R e=90,000$ is close to experiment. The noise levels produced by the lower Reynolds number results are higher.
The computations that use turbulence models at $R e=$ 90, 000 produce inconsistent results. This is likely due to the onset of transition near the body since the chosen Reynolds number is very near the supercritical range. It is believed that these difficulties will persist until CFD has the ability to accurately predict transition.

At a Reynolds number of 5 million, results using three different turbulence models give self-consistent results for far-field noise. Also, the mean drag is in good agreement with experimental results, and the increased Strouhal number of about 0.3 reflects an increase similar to that seen in experiments. At this transcritical Reynolds number, the computed flow transitions near the stagnation point on the cylinder, so the boundary layer is fully turbulent by the time it separates. Therefore, it appears that current state-of-the-art CFD may be best suited for predicting unsteady circular cylinder flows (and their resulting far field noise) that are far outside, either well below or above, the supercritical regime.

\section{ACKNOWLEDGEMENTS}

The authors wish to thank R. Biedron of NASA Langley Research Center for his helpful discussion and advice during the course of this investigation. Research done by the first author is a portion of what will be included in a Master of Science Thesis with The George Washington University. The first author wishes to acknowledge support from the NASA Langley Research Center, Aeroacoustics Branch, for this research.

\section{References}

1. Morkovin, M. V., "Flow Around a Circular Cylinder A Kaleidoscope of Challenging Fluid Phenomena," $A S M E$ Symposium on Fully Separated Flows, 1964, pp. 102-118.

2. Zdravkovich, M. M., "Conceptual Overview of Laminar and Turbulent Flows Past Smooth and Rough Circular Cylinders," Journal of Wind Engineering and Industrial Aerodynamics, Vol. 33, 1990, pp. 53-62.

3. Roshko, A., "Experiments on the Flow Past a Circular Cylinder at Very High Reynolds Number," Journal of Fluid Mechanics, Vol. 10, No. 2, 1961, pp. 345-356.

4. Achenbach, E., and Heinecke, E., "On Vortex Shedding from Smooth and Rough Cylinders in the Range of Reynolds Numbers $6 \times 10^{3}$ to $5 \times 10^{6}$," Journal of Fluid Mechanics, Vol. 109, 1981, pp. 239-251.

5. Schlichting, H., Boundary-Layer Theory, McGraw-Hill Series in Mechanical Engineering, McGraw-Hill, New York, Seventh edition, 1979. Translated by J. Kestin.

6. Blackburn, H. M., and Melbourne, W. H., "The Effect of Free-Stream Turbulence on Sectional Lift Forces on a Circular Cylinder," Journal of Fluid Mechanics, Vol. 306, 1996, pp. 267-292.

7. Alemdarō̄lu, N., Rebillat, J. C., and Goethals, R., "An Aeroacoustic Coherence Function Method Applied to Circular Cylinder Flows," Journal of Sound and Vibration, Vol. 69, No. 3, 1980, pp. 427-439.

8. Mittal, R., and Balachandar, S., "Effect of ThreeDimensionality on the Lift and Drag of Nominally Two- 
Dimensional Cylinders," Physics of Fluids, Vol. 7, No. 8, Aug. 1995, pp. 1841-1865.

9. Belov, A., Jameson, A., and Martinelli, L., "ThreeDimensional Unsteady Incompressible Flow Calculations Using Multigrid," AIAA paper 97-0443, Jan. 1997.

10. Franke, R., Rodi, W., and Schonung, B., "Numerical Calculation of Laminar Vortex-Shedding Flow Past Cylinders," Journal of Wind Engineering and Industrial Aerodynamics, Vol. 35, 1990, pp. 237-257.

11. Visbal, M. R., "Evaluation of an Implicit Navier-Stokes Solver for Some Unsteady Separated Flows," AIAA Paper 86-1053, May 1986.

12. Rumsey, C. L., Thomas, J. L., Warren, G. P., and Liu, G. C., "Upwind Navier-Stokes Solutions for Separated Periodic Flows," AIAA Journal, Vol. 25, No. 4, 1987, pp. 535541.

13. Braza, M., Chassaing, P., and Ha Minh, H., "Numerical Study and Physical Analysis of the Pressure and Velocity Fields in the Near Wake of a Circular Cylinder," Journal of Fluid Mechanics, Vol. 165, 1986, pp. 79-130.

14. Lecointe, Y., and Piquet, J., "On the Use of Several Compact Methods for the Study of Unsteady Incompressible Viscous Flow Round a Circular Cylinder," Computers and Flu$i d s$, Vol. 12, No. 4, 1984, pp. 255-280.

15. Farassat, F., and Succi, G. P., "The Prediction of Helicopter Discrete Frequency Noise," Vertica, Vol. 7, No. 4, 1983, pp. 309-320.

16. Revell, J. D., Prydz, R. A., and Hays, A. P., "Experimental Study of Airframe Noise vs. Drag Relationship for Circular Cylinders," Lockheed Report 28074, Feb. 1977. Final Report for NASA Contract NAS1-14403.

17. Brentner, K. S., Cox, J. S., Rumsey, C. L., and Younis, B. A., "Computation of Sound Generated by Flow Over a Circular Cylinder: An Acoustic Analogy Approach," Proceedings of the Second Computational Aeroacoustics Workshop on Benchmark Problems, 1997. To appear as a NASA $\mathrm{CP}$.

18. Rumsey, C. L., Sanetrik, M. D., Biedron, R. T., Melson, N. D., and Parlette, E. B., "Efficiency and Accuracy of TimeAccurate Turbulent Navier-Stokes Computations," Computers and Fluids, Vol. 25, No. 2, 1996, pp. 217-236.

19. Roe, P., "Approximate Riemann solvers, parameter vectors, and difference schemes," Journal of Computational Physics, Vol. 43, 1981, p. 357.

20. Spalart, P. R., and Allmaras, S. R., "A One-Equation Turbulence Model for Aerodynamic Flows," La Recherche Aerospatiale, , No. 1, 1994, pp. 5-21.

21. Menter, F. R., "Improved Two-Equation $k-\omega$ Turbulence Models for Aerodynamic Flows," NASA TM 103975, Oct. 1992.

22. Abid, R., "Evaluation of Two-Equation Turbulence Models for Predicting Transitional Flows," Int. J. Eng. Turbulence, Vol. 31, 1993, pp. 831-840.
23. Menter, F. R., and Rumsey, C. L., "Assessment of TwoEquation Turbulence Models for Transonic Flows," AIAA Paper 94-2343, 1994.

24. Jones, W. P., and Launder, B. E., "The Calculation of LowReynolds-Number Phenomena with a Two-Equation Model of Turbulence," Int. J. Heat Mass Transfer, Vol. 16, 1973, pp. 1119-1130.

25. Przulj, V., and Younis, B. A., "Some Aspects of the Prediction of Turbulent Vortex Shedding from Bluff Bodies," Symposium on Unsteady Separated Flows, 1993 ASME Fluids Engineering Division Annual Summer Meeting, Vol. 149, Washington, DC, 1993.

26. Anderson, W. K., and Batina, J. T., "Accurate Solutions, Parameter Studies, and Comparisons for the Euler and Potential Flow Equations," AGARD 62nd Meeting of the Fluid Dynamics Panel Symposium, AGARD-CP-437, 1988, pp. $15-1-15-16$.

27. Brentner, K. S., "Prediction of Helicopter Discrete Frequency Rotor Noise-A Computer Program Incorporating Realistic Blade Motions and Advanced Formulation," NASA TM 87721, Oct. 1986.

28. Ffowcs Williams, J. E., and Hawkings, D. L., "Sound Generated by Turbulence and Surfaces in Arbitrary Motion," Philosophical Transactions of the Royal Society, Vol. A264, No. 1151, 1969, pp. 321-342.

29. Kacker, S. C., Pennington, B., and Hill, R. S., "Fluctuating Lift Coefficient For a Circular Cylinder in Cross Flow," Journal of Mechanical Engineering Science, Vol. 16, No. 4, 1974, pp. 215-224.

30. Szepessy, S., and Bearman, P. W., "Aspect ratio and end plate effects on vortex shedding from a circular cylinder," Journal of Fluid Mechanics, Vol. 234, 1992, pp. 191-217.

31. Van Dyke, M., An Album of Fluid Motion, The Parabolic Press, Stanford, California, 1982.

32. Cantwell, B., and Coles, D., "An experimental study of entrainment and transport in the turbulent near wake of a circular cylinder," Journal of Fluid Mechanics, Vol. 136, 1983, pp. 321-374.

33. Shang, J. S., "Oscillatory Compressible Flow Around a Cylinder," AIAA Paper 82-0098, Jan. 1982.

34. Pulliam, T., "Introduction of Chaos Theory in CFD," AIAA Paper 90-1440, June 1990.

35. West, G. S., and Apelt, C. J., "Measurement of Fluctuating Effects on a Circular Cylinder in a Uniform Flow at Sub-Critical Reynolds Numbers. Part 1, The Pressure Distribution and Lift and Drag Forces on an Elemental Cross Section," Department of Civil Engineering Research Report CE109, The University of Queensland, July 1990. 